\title{
LA FORMATION DES INTERPRÈTES POUR LEURS INTERVENTIONS EN SANTÉ MENTALE : DÉVELOPPEMENT D’UN DOSSIER PÉDAGOGIQUE
}

\author{
Orest Weber, Florence Faucherre
}

Orspere-Samdarra | « Rhizome »

2020/1 N 75-76 | pages 99 à 109

ISSN 1622-2032

Article disponible en ligne à l'adresse :

https://www.cairn.info/revue-rhizome-2020-1-page-99.htm

Distribution électronique Cairn.info pour Orspere-Samdarra.

(C) Orspere-Samdarra. Tous droits réservés pour tous pays.

La reproduction ou représentation de cet article, notamment par photocopie, n'est autorisée que dans les limites des conditions générales d'utilisation du site ou, le cas échéant, des conditions générales de la licence souscrite par votre établissement. Toute autre reproduction ou représentation, en tout ou partie, sous quelque forme et de quelque manière que ce soit, est interdite sauf accord préalable et écrit de l'éditeur, en dehors des cas prévus par la législation en vigueur en France. Il est précisé que son stockage dans une base de données est également interdit. 
Orest Weber

Unité Psy et Migrants

Département de psychiatrie CHUV

Lausanne
Florence Faucherre

Unité Psy et Migrants

Département de psychiatrie

CHUV

Lausanne

LA FORMATION DES INTERPRÈTES
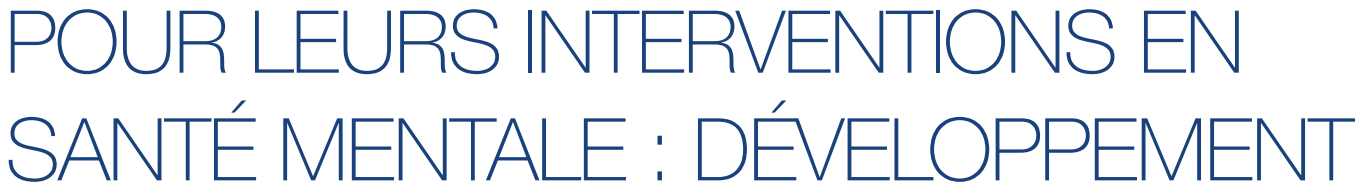

D'UN DOSSIER PÉDAGOGIQUE

Dans les pays d'Europe occidentale, les institutions de santé mentale prennent en charge un nombre croissant de patients migrants ne parlant pas ou peu la langue locale. Si elles veulent éviter des discriminations linguistiques concernant l'accessibilité et la qualité des soins, ces institutions n'ont généralement d'autre choix que de recourir à des tiers assurant l'interprétariat. S'agissant du profil de ces tiers, la littérature souligne l'importance, plus encore en psychiatrie qu'ailleurs, de confier la médiation linguistique et culturelle à des professionnel|le's dûment formé'e·s (Bauer et Alegria, 2010).

Si dans d'autres pays francophones on tend à appeler interprètes de services publics les professionnel.le's de l'interprétariat intervenant dans la santé, il en va autrement de la Suisse romande, cadre géographique de notre projet, où la désignation consacrée est interprète communautaire. Grâce à diverses instances fédérales et associatives, ces interprètes ont, dans notre pays, la possibilité d'acquérir un certificat et un brevet reconnus au plan fédéral'1. Dans les formations conduisant à ces titres, le travail dans la santé mentale fait l'objet d'enseignements spécifiques. Pour leur part, les clinicien'ne's travaillant dans la santé mentale disposent également d'espaces de formation en nombre croissant pour s'initier ou se perfectionner dans le travail avec les interprètes.

L'objectif des formations préparant les professionnelle's de la santé et de 
de connaissances, d'aptitudes et d'attitudes qui favorisent des pratiques fonctionnelles au regard des buts des entretiens cliniques. Dans la santé mentale, l'un des principaux enjeux pour les clinicien'ne's et les interprètes communautaires réside dans la nécessité permanente de moduler et d'ajuster leurs pratiques respectives (Bot, 2005; Delizée et de Ridder, 2016). La multitude de dispositifs et d'activités constitutives de la psychiatrie contemporaine confronte en effet les triades patient·es-interprètes-clinicien'ne's à des défis diversifiés. Afin de contribuer à une meilleure connaissance de ces défis, l'équipe de recherche de notre unité de psychiatrie transculturelle (composé d'un linguiste, de deux psychiatres et d'une psychologue) a décidé d'étudier les difficultés qui prédominent dans les consultations d'investigation psychiatriques ou psychologiques. L'objectif était d'emblée de contribuer, sur cette base, aux formations des interprètes et des clinicien'ne.s.

Les consultations d'investigation ne sont pas seulement importantes pour l'accès aux soins des migrant.e.s. La littérature (Farooq et al., 1997; Guex et Singy, 2003 ; Brisset et al., 2013), de même que nos observations initiales effectuées sur le terrain (Weber et al., 2017) montrent qu'elles sont particulièrement délicates pour les professionnelle's qui doivent y intervenir en triade. Plusieurs recherches observationnelles questionnent, d'une part, la qualité des pratiques des professionnel|le's - et tout particulièrement des interprètes - engagé'e's dans les investigations triadiques (Farooq et al., 1997). D'autre part, les processus d'investigation apparaissent particulièrement délicats sur le plan relationnel. Les personnes impliquées dans les rencontres doivent en effet souvent apprendre à se connaître et à se faire confiance dans un contexte où les clinicien'ne·s craignent de perdre des indices diagnostics essentiels en raison d'une médiation linguistique insuffisamment rigoureuse. D'importantes inquiétudes et incertitudes sont donc toujours à attendre chez les professionnel|le·s, de même que des tensions liées à des questions de contrôle et de pouvoir (Guex et Singy, 2003; Brisset et al., 2013).

Pour promouvoir des pratiques et stratégies utiles aux interprètes, clinicien'ne's et patient.ess en interaction lors d'investigations psychiatriques, nous avons filmé et analysé des entretiens cliniques. Conformément aux principes d'une recherche-action (Nikodemus et Swabey, 2016), nous avons ensuite conçu et testé des interventions pédagogiques, puis réalisé et mis en ligne un dossier

$\underline{2}$ Vous pouvez le consulter sur cette page www.hospitals4equity.ch pédagogique à destination de formateurs'trice's et d'interprètes en autodidacte $^{2}$. Un dossier similaire en lien avec les formations pour clinicien'ne's est en préparation. 
L'objectif de cet article est de présenter et d'illustrer le dossier pédagogique réalisé, produit essentiel de notre projet dont nous espérons ainsi favoriser la diffusion. En préalable, le processus scientifique et didactique ayant permis sa création sera brièvement rapporté. Des indications méthodologiques supplémentaires et les références relatives au plan procédural de notre enquête sont disponibles dans une publication antérieure (Weber et al., 2017).

\section{L'analyse pluridisciplinaire de consultations}

L'analyse de consultations a principalement cherché à comprendre comment les interventions des interprètes et la collaboration interprofessionnelle pourraient être améliorées lors d'investigations psychiatriques triadiques. Compte tenu de la composition de l'équipe de notre unité, nous avons opté pour une étude interdisciplinaire combinant une approche clinique et une approche discursive. La recherche repose sur des enregistrements vidéo de consultations d'investigation triadiques, transcrites et traduites par un autre interprète. Les vidéos ont ensuite été soumises à une analyse clinique répertoriant toutes les séquences au sein desquelles les restitutions par les interprètes ou la collaboration entre interprète et thérapeute compliquent ou limitent le travail d'investigation. Les séquences identifiées ont fait l'objet d'une analyse linguistique s'intéressant principalement : aux prises de parole, interruptions, etc; ; aux activités de prévention et de réparation de malentendus; à la cohérence et pertinence des propos originaux et des restitutions; et à la manière dont les personnes gèrent de possibles tensions relationnelles dans les échanges (gestion des faces).

Suite à cette double analyse, des hypothèses articulant les observations cliniques et linguistiques ont été formulées. Elles feront l'objet d'une publication plus extensive. Ces hypothèses se rapportent principalement à six défis concernant tantôt les deux catégories de professionnel·le·s, tantôt les interprètes seulement. Ces défis sont synthétisés ci-dessous dans un langage profane et uniquement sous l'angle du travail des interprètes, car ce sont eux. elles qui sont au centre du matériel didactique présenté par la suite. Notre analyse met en lumière que, lorsqu'il.elle.s sont confronté.e.s à ces défis, les professionnel|le·s sélectionnent certaines options et en écartent d'autres. Ces options - souvent prises inconsciemment - peuvent avoir des conséquences plus ou moins souhaitables du point de vue clinique. On peut donc parler de ces défis en termes d'embranchements interactionnels cliniquement sensibles et c'est là ce qui fait tout leur intérêt en vue de formations. 


\section{Six défis pour les interprètes}

\section{Propos difficiles à comprendre (explications ou récits confus, réponses hors sujet, néologismes, etc.)}

Les interprètes sont souvent confrontés à des discours dont les liens logiques internes, de même que les liens avec le contexte interactionnel plus large (par exemple, celui d'une réponse avec la question précédente) sont difficiles à comprendre. Une autre difficulté émane de néologismes. Les interprètes peuvent alors restituer ces discours, généralement énoncés par les patient·e·s, de manière plus facilement compréhensible ou utilisable pour les clinicien.ne·s. Cela prive toutefois les psychiatres/psychologues d'informations décisives, notamment sur une possible désorganisation du discours et de la pensée chez lea patiente.e. Comme alternative, les interprètes peuvent métacommuniquer sur leurs difficultés de compréhension et/ou de restitution puis se limiter à une restitution d'éléments ou thèmes qu'ils sont sûr d'avoir compris. Une autre option réside dans un interprétariat en semi-simultané (chuchotage).

\section{Mentions d'émotions et de perceptions subjectives}

Lorsque les propos échangés contiennent des mentions d'émotions et de perceptions subjectives (par exemple, le sentiment que quelque chose préoccupe l'autre), les clinicien'ne's expriment un besoin de restitutions particulièrement précises. Ce besoin de précision porte tant sur les propos de patiente's parlant d'eux. elles-mêmes, que sur les interventions des clinicien'ne·s, notamment lorsqu'ils·elles investiguent le vécu des patientees ou livrent leur vision de la situation en faisant référence à des émotions ou perceptions. Les échanges métacommunicatifs entre professionnel.le's apparaissent particulièrement importants si les propos traitant d'émotions ou d'éléments relevant du vécu subjectif mettent les interprètes en difficulté (un mot pour une émotion n'a pas d'équivalent direct dans l'autre langue; une formulation subjective du.de la clinicien'ne est très compliquée et semble incomprise par le.a patiente ; etc.).

\section{Propos sensibles en termes relationnels}

Certains propos de clinicien'ne's et de patientee's peuvent être perçus par les interprètes comme susceptibles de créer ou renforcer une tension relationnelle au sein de la triade. Les interprètes ont alors soit l'option de livrer des restitutions avec une charge conflictuelle similaire, soit celle d'atténuer cette charge par de subtiles modifications 
du sens. La seconde de ces options est problématisée par les clinicien·ne·s, craignant de se voir privé.e.s d'éléments importants concernant tant le fonctionnement relationnel du.de la patient.e que l'évolution de la relation thérapeutique.

\section{Interventions conclusives de clinicien·ne·s (propos hors alternance question/réponse)}

Avant d'introduire une nouvelle question, les clinicien'ne's qui viennent d'entendre la restitution d'une réponse ou d'un récit d'un'e patientee reprennent souvent très brièvement la parole. Ces interventions conclusives, qui sont une sorte d'ajout au schéma question-réponse dominant au sein des entretiens, commentent ce qui vient d'être dit ou représentent de brèves prises de position de la part du·de la clinicien.ne. Ces interventions brèves sont parfois importantes sur les plans clinique et relationnel. Elles sont parfois restituées, mais à d'autres occasions, elles sont omises.

\section{Interruptions de l'interprète en cours de restitution}

Les interprètes sont souvent confronté.e.s à des interruptions par l'une des deux autres parties alors qu'ils'elles sont encore en train de restituer ce qui a été dit précédemment. II s'agit alors de retenir tous les nouveaux éléments énoncés, de même que tous les éléments encore non restitués. Certains interprètes ont des facultés impressionnantes de mémorisation dans ces situations, mais elles conduisent, malgré tout, souvent à d'importantes pertes d'informations cliniques, de même qu'à des réorganisations massives des propos pouvant par exemple gommer des problèmes de cohérence dans les discours originaux (cf. le premier défi). Des échanges métacommunicatifs entre clinicien'ne et interprète peuvent s'avérer essentiels dans ces cas.

\section{Éléments non verbaux}

Les gestes à portée communicationnelle des patient·e's et des clinicien'ne's sont parfois compréhensibles sans traduction, mais de loin pas systématiquement. Les codes non verbaux connaissent en effet aussi de grandes variations d'une communauté linguistique à l'autre. Les interprètes ont donc la difficile tâche d'identifier les situations où il est utile qu'ils'elles interviennent activement sur les gestes communicationnels échangés, puis de choisir une modalité de restitution : explicitation verbale du geste ou substitution par un geste équivalent, mais compréhensible pour l'autre. 


\section{Mise en œuvre des résultats dans des formations}

Parallèlement à l'achèvement du travail d'analyse, nous avons commencé à utiliser les résultats et les extraits d'entretien dans diverses activités de formation. Le premier auteur a en particulier été sollicité par l'association Appartenances pour intervenir dans la formation continue des interprètes communautaires dans le cadre du module "Interprétariat dans le domaine de la santé psychique " au sein des cours centrés sur les techniques d'interprétation et la gestion pratique de propos particulièrement difficiles à restituer. Le cours élaboré dans ce cadre est d'une durée de trois heures et demie et a été donné à trois reprises sur les quatre dernières années, ce qui a permis de l'adapter progressivement en bénéficiant des retours et idées des participant.es et de l'organisatrice de la formation. Au vu de l'intérêt marqué et de la satisfaction que le cours suscite de toutes parts, nous avons jugé opportun de réaliser un dossier pédagogique pouvant être utilisé par d'autres formateur.rice.s.

\section{Dossier pédagogique pour la formation des interprètes}

Le dossier pédagogique élaboré pour les formations d'interprètes est un support pour des cours de spécialisation ou d'approfondissement dans le domaine de l'interprétariat en santé mentale, en particulier dans un contexte d'investigation. Dans sa présentation, il est prioritairement orienté vers un enseignement présentiel articulant des discussions réflexives en groupe et des moments d'expérimentation pratique individuelle et de prise de notes. Le matériel est toutefois conçu de sorte à permettre également une utilisation en autodidacte.

Les objectifs de formations suivants peuvent être atteints dans des cours basés sur ce dossier :

- rendre les participante's plus conscients de certains défis clés rencontrés lors d'interventions dans le champ de la santé mentale, en particulier lors d'entretiens d'investigation;

- renforcer les capacités des participant.e.s à reconnaître :

- des aspects du discours importants pour les clinicien'ne's et nécessitant une précision particulière lors des restitutions et à adapter leur pratique dans ce sens;

- des éléments méritant d'être thématisés dans le cadre d'une métacommunication et à réaliser cette métacommunication.

L'organisation du dossier part de quatre extraits qui permettent chacun d'aborder un ou plusieurs des défis susmentionnés. Pour chaque extrait, le matériel contient 
six documents : une introduction aux défis présents dans l'extrait; l'extrait précédé de la question de départ pour la discussion réflexive et d'éléments de contexte; une liste de questions d'approfondissement à poser aux participant.e.s; une fiche de travail permettant aux apprenant·e's de noter leurs observations (leçons tirées et stratégies possibles); et un commentaire de l'extrait contenant également une liste des leçons et stratégies pouvant être suggérées aux participant·es.

Pour illustration, voici la reproduction des deux éléments clés des pages consacrées au premier des quatre extraits du dossier. II s'agit de l'extrait lui-même (accompagné de la question initiale et des éléments de contexte) et du commentaire de l'extrait. L'extrait 1 est centré sur les défis 2, 3 et 4 : les mentions d'émotions et de perceptions subjectives; les propos sensibles en termes relationnels; et les interventions conclusives de clinicien.ne's (propos hors alternance question/réponse).

\section{Extrait 1 : question de départ, contexte et transcription ${ }^{3}$}

\section{Questions de départ pour la discussion/réflexion}

Quels sont les défis pour l'interprète dans ce passage? Comment les relève-t-elle? Quelles bonnes stratégies sont utilisées ou pourraient l'être?

\section{Contexte}

Cet extrait est tiré du troisième entretien d'investigation du patient avec cette clinicienne; le début de l'entretien est consacré aux grandes lignes de l'évolution de la situation et des symptômes depuis la dernière consultation. Le divorce du patient est le premier grand sujet qui est introduit par la thérapeute et fera l'objet d'une bonne partie de l'entretien; le patient avait préalablement évoqué ce divorce, mais n'en avait jamais parlé en détail avec la clinicienne. Voici comment

3 Dans la transcription de l'extrait 1, le texte original en français est rendu par des caractères romains, celui dans la langue du patient (dari) par des caractères italiques. Un volume sonore bas est signalé par la convention ${ }^{\circ}$ lerifsoitj ${ }^{\circ}$ et des discours simultanés (chevauchements) par la convention [skkfi] [rotoi]. $C$ renvoie à la clinicienne, I, à l'interprète et P, au patient. le sujet est introduit et quelle discussion cette introduction déclenche entre les participante.s :

1. C : Mhm. En tout cas, j'ai le sentiment que c'est quelque chose qui vous préoccupe beaucoup, parce

2. que vous l'avez souvent mentionné, sans en parler plus. Donc je me dis que [peut-être]

3. I: [Elle dit]

4. C : ${ }^{\circ} \mathrm{C}$ a vous occupe beaucoup ${ }^{\circ}$

5. I : De toute façon, je vois que vous êtes très préoccupé par ça, chaque fois que vous parlez de ça 
6. Vous êtes triste, sans que vous expliquiez davantage.

7. $\quad \mathrm{P}$ : Eh ben, si personne ne vous pose la question

8. $\quad 1:$ [Ouais]

9. $\mathrm{P}:$ [On ne peut pas] répondre comme ça au tac au tac

10. I : Quand on me pose pas la question, je peux pas répondre euh sans question

11. C : Mhm, bien sûr... Vous avez été marié longtemps?

12. I : Ça fait longtemps que vous vous êtes marié?

\section{Extrait 1 : commentaire de l'extrait}

\section{Les termes utilisés pour parler du vécu subjectif}

Nous avons ici un exemple d'investigation des émotions du patient. La clinicienne entame la discussion en expliquant, à la ligne 1, qu'elle sent de la préoccupation chez le patient au sujet de son divorce. Cet élément est repris par l'interprète (ligne 5), mais celle-ci ajoute également un complément (ligne 6) qui n'est pas présent dans le discours de la thérapeute et qui exprime une émotion précise : la tristesse.

II est possible que l'interprète ait ajouté cet élément afin que le patient comprenne mieux que la clinicienne s'intéresse à ses émotions. Mais cette manière de traduire peut avoir une conséquence problématique (qui n'est pas observable ici) : si le patient parle de tristesse, la clinicienne pensera qu'il le fait spontanément, alors qu'en réalité, c'est l'interprète qui lui a mis ce mot dans la bouche. Les termes précis utilisés de part et d'autre pour les émotions sont souvent décisifs pour un travail efficace en psychiatrie et psychothérapie.

Un autre défi pour l'interprète est lié aux termes utilisés par la thérapeute pour parler de son propre vécu subjectif en lien avec la situation du patient: "j'ai le sentiment que ", à la ligne 1, ainsi que " je me dis que peut-être ", à la ligne 2.

Selon nos observations ces éléments sont souvent transmis au patient de manière moins nuancée (ici : " de toute façon je vois que ", à la ligne 5), ce qui ne permet pas au patient d'avoir accès au caractère hypothétique de ce que dit la clinicienne. Pour pouvoir passer à la traduction, de tels éléments, qui peuvent facilement passer inaperçus, demandent une attention particulière de la part des interprètes. Mais, pour les cliniciens, ces éléments paraissent en général moins importants que les mots pour parler des émotions des patients. 


\section{Leçons à retenir}

- Les clinicien'ne's en santé mentale ont besoin d'informations aussi précises que possible sur les émotions et le vécu subjectif des patient·e.s.

- La précision de la restitution des questions des clinicien'ne's est très importante pour que les clinicien'ne's sachent exactement à quoi les patient.e's répondent.

\section{Stratégies}

- Se concentrer particulièrement sur la mémorisation des termes et expressions portant sur les émotions.

- Métacommuniquer avec le.a clinicien'ne en cas de questions trop longues ou complexes pour permettre une traduction précise et, si nécessaire, demander de reformuler la question plus simplement.

\section{Les interjections hors question-réponse avec une portée relationnelle sensible}

Dans la situation présente, on assiste à une sorte de négociation des responsabilités entre la clinicienne et le patient. Elle est déclenchée par la clinicienne qui dit au patient qu'il n'a pour l'instant presque pas abordé le sujet de son divorce avec elle. La réaction du patient suggère qu'il a interprété cette remarque comme une critique. Le patient s'engage, en effet, dans une explication quant aux raisons de l'absence de tout approfondissement de ce thème dans les rencontres précédentes: la clinicienne ne lui a pas posé la question. On se trouve donc ici dans un moment relationnel délicat du type " ce n'est pas de ma faute, c'est de la vôtre".

Dans ce contexte, le fait que la thérapeute réagisse en disant "bien sûr " (ligne 11) revêt une importance particulière : elle signale par là qu'elle valide le point de vue défendu par le patient. Ce feed-back n'est pas repris par l'interprète à la ligne 12, qui traduit simplement la question posée au patient. On observe, de manière générale, que les éléments qui relèvent des questions et réponses sont souvent privilégiés, lors de la traduction, par rapport au reste du contenu du discours. Les questions et les réponses représentent des éléments essentiels du contenu des échanges, mais le travail de traduction ne peut se résumer à ces éléments, surtout dans un contexte clinique où le discours dans son ensemble a une importance primordiale pour la relation et le travail thérapeutique. Les interjections hors question-réponse sont des éléments qui peuvent facilement passer inaperçus, mais peuvent avoir une grande importance dans certaines situations. 


\section{Leçons à retenir}

- Les propos qui ne font partie ni d'une question ni d'une réponse risquent particulièrement d'être oubliés à la traduction.

- Parfois ces propos peuvent paraître insignifiants, mais être importants, par exemple, parce qu'ils interviennent dans des moments relationnels délicats.

\section{Stratégies possibles}

- Être attentif aux propos qui ne font partie ni d'une question ni d'une réponse.

- Observer si des tensions apparaissent et soigner tout particulièrement la précision de la traduction des propos qui renforcent ou apaisent la tension.

- Traduire des remarques conclusives courtes immédiatement, avant que le·a clincien'ne ne pose la prochaine question.

- Métacommuniquer avec le·a clinicien'ne pour demander son accord de principe pour une traduction en semi-simultané (chuchotage) de remarques conclusives courtes si le temps pour une traduction en consécutive devait manquer, puis pratiquer de la sorte.

\section{En guise de conclusion}

Notre expérience lors des formations d'interprètes montre que les discussions déclenchées par des extraits tels que celui présenté ici sont animées et riches. II est généralement possible d'aider les participantees à formuler eux'ellesmêmes une part importante des idées, leçons et stratégies contenues dans le commentaire ci-dessus. II est frappant que les interprètes entament souvent la formation en ayant déjà certaines idées sur ce que l'on attend d'eux dans la santé mentale, notamment des restitutions reproduisant au plus près les propos originaux sans tenter de les rendre plus compréhensibles pour le destinataire. Ils n'en trouvent cependant que plus utile de regarder de près des échanges réels et de réfléchir, sur cette base, à la manière dont on peut exercer son métier avec conscience professionnelle, mais sans culpabilité indue face à un tel horizon d'attentes.

Pour soutenir l'impact effectif des réflexions sur les pratiques - et, par conséquent, sur la qualité du travail des interprètes et leur confort - nous proposons, dans les cours, autant d'exercices pratiques que possible (test de stratégies, jeux de rôles). Les participantees partent aussi du cours avec pour mission d'utiliser ce qu'ils'elles ont appris dans les interventions suivantes en psychiatrie et d'en reparler avec leurs collègues lors des prochains cours. 
4 Nous tenons à

remercier Argyro Daliani, Esther-Amélie Diserens, Florian Chmetz, Melissa Riat, Cati Paiva Pinheiro, Vincent Wenger, Jonathan Klemp et Christopher Hasler pour leurs contributions à l'étude et/ou à l'élaboration du dossier didactique. Notre gratitude va également à l'association Appartenances (secteur interprétariat) pour sa collaboration précieuse tant pour la production des données que pour le test des formations d'interprètes. Nous tenons enfin à remercier l'Office fédéral de la santé publique pour le soutien financier.

On notera, pour conclure, que l'une des principales forces de notre formation - son focus clair sur la précision des restitutions dans des domaines essentiels du point de vue de la clinique en santé mentale - représente également sa principale limite. Dans nos cours, le regard reste en effet largement rivé sur la mission la plus centrale et consensuelle incombant aux interprètes : la médiation linguistique (et gestuelle). La seule autre activité que nous thématisons avec insistance est la métacommunication, qui permet aux interprètes de parler de leurs difficultés pour en informer le.la clinicien'ne et/ou pour discuter de solutions possibles avec eux. elles (par exemple, le chuchotage). Or l'on sait bien aujourd'hui que les activités des interprètes sur les terrains de santé mentale sont bien plus diversifiées dans les faits (Bot, 2005; Delizée et De Ridder, 2016), dépassant largement ces deux activités. Une formation telle que la nôtre n'est donc en aucun cas suffisante pour préparer les interprètes à leurs interventions en psychiatrie et d'autres apports sont absolument nécessaires. On pense, par exemple, à des échanges d'informations entre clinicien'ne's et interprètes à propos des challenges relationnels, émotionnels et culturels possibles avec certains types de patiente's (par exemple au sujet des psychotiques décompensés, des personnes suicidaires, etc.), mais également à un travail réflexif à propos de tout le large spectre des tâches et missions que les interprètes peuvent réaliser en psychiatrie (accompagnement dans des services sociaux, contribution au diagnostic, apport général d'informations contextuelles/culturelles, etc.) ${ }^{4}$. D

\section{BIBLIOGRAPHE}

Bauer, A. M., et Alegría, M. (2010). Impact of patient language proficiency and interpreter service use on the quality of psychiatric care: A systematic review. Psychiatric Services, 61(8), 765-773.

Bot, H. (2005). Dialogue interpreting in mental health. Amsterdam: Rodopi.

Brisset, C., Leanza, Y. et Laforest, K. (2013). Working with interpreters in health care: a systematic review and meta-ethnography of qualitative studies. Patient Éducation et Counseling, 91(2), 131-140.
Delizée, A., et De Ridder, P. (2016). L'interprètekaléidoscope ou le questionnement identitaire de l'interprète en santé mentale. Un état de la question. Mons, Belgique : Université de Mons.

Farooq, S., Fear, C. F. et Oyebode, F. (1997). An investigation of the adequacy of psychiatric interviews conducted through an interpreter. Psychiatric Bulletin, 21(4), 209-213.

Guex, P. et Singy, P. (dir.). (2003). Quand la médecine a besoin d'interprètes. Genève : Médecine et Hygiène.
Nicodemus, B., et Swabey, L. (2016). Action research. Dans C. V. Angelelli et B. J. Baer (Eds.), Researching translation and interpreting (p. 157-167). London: Routledge.

Weber, O., Daliani, A., Diserens, E.-A., Chmetz, F. et Faucherre, F. (2017). La recherche interdisciplinaire sur l'interprétariat dans les services publics: expériences tirées d'une étude exploratoire dans le cadre d'investigations psychiatriques. Dragoman Journal of Translation Studies, 5(7), 38-59. 